\title{
De quelques problèmes d'une éducation civique : que fait-on quand on cherche à élucider des principes?
}

\section{Frédéric François}

\section{(2) OpenEdition \\ Journals \\ Édition électronique \\ URL : http://journals.openedition.org/trema/1691 \\ DOI : 10.4000/trema.1691 \\ ISSN : 2107-0997 \\ Éditeur \\ Faculté d'Éducation de l'université de Montpellier}

Édition imprimée

Date de publication : 1 octobre 1999

Pagination : 27-40

ISSN : 1167-315X

\section{Référence électronique}

Frédéric François, «De quelques problèmes d'une éducation civique : que fait-on quand on cherche à élucider des principes? », Tréma [En ligne], 15-16| 1999, mis en ligne le 01 octobre 1999, consulté le 19 avril 2019. URL : http://journals.openedition.org/trema/1691 ; DOI : 10.4000/trema.1691

Ce document a été généré automatiquement le 19 avril 2019.

Trema 


\title{
De quelques problèmes d'une éducation civique : que fait-on quand on cherche à élucider des principes?
}

\author{
Frédéric François
}

1 Plus sans doute que dans les autres matières, le problème de ce à quoi sert l'enseignant est ici troublant. Certes l'enseignant sait des faits, connaît des penseurs que les élèves ne connaissent pas. Mais il n'est pas en situation de leur dire : «je connais les principes selon lesquels la vie en société doit être vécue, apprenez-les et tout ira bien » non plus que : «apprenez à penser : suivez mon exemple».

2 Et d'abord, il ne s'agit pas que de parler : les deux énoncés qui servent de titre ne sont pas équivalents. Quelle relation entre le développement d'une certaine socialité, une façon pacifiée de vivre en commun et la prise de conscience de ce que c'est qu'être plus spécifiquement "citoyen»? Quel lien de cela aux savoirs sur l'histoire, sur les constitutions? Avec la possibilité d'argumenter, de comprendre, de réfuter? Avec la position explicite et réfléchie (ce n'est pas forcément la même chose) des règles et des principes de la vie en société?

3 Un tel faisceau de questions est complexe. On n'a pas la prétention d'apporter la "vraie » réponse. Plutôt de présenter quelques fils de réflexion concernant le rôle spécifique de l'institution scolaire dans la « fabrication » de citoyens.

4 Certes, l'institution scolaire est une " agence de socialisation » spécifique. Directement par la vie en commun qu'elle suppose, indirectement par les tâches qu'elle impose et par la culture qu'elle apporte. En même temps personne ne peut penser que l'école est le lieu exclusif de cette socialisation ni, corrélativement, que ce serait sa seule fonction.

5 On peut aussi noter que la formulation titre qui est proposée à ce colloque, regroupant savoir, langages et citoyenneté, est comme toute formulation titre, forcément discutable. Pour commencer par les deux premiers mots : savoir au singulier et langages au pluriel? 
Comprendre, réfléchir, discuter sont-ils d'un côté, de l'autre, ailleurs ? Même s'il est vrai qu'il faut avoir des savoirs pour que le proche devienne objet de réflexion et que cela passe par du langage.

6 Une autre direction serait de se demander si les problèmes de notre époque sont très différents de ce qu'ils étaient autrefois. Il y a certainement, en particulier en ce qui concerne l'évolution technique, la «mondialisation», la télévision des différences considérables. A tort ou à raison, nous percevons les époques passées comme plus unanimes, transmettant plus simplement des « valeurs » ou des « principes » que la notre où les problèmes seraient plus complexes et la diversité plus grande. C'est en partie vrai, sans doute, en même temps, il me semble que dans ce sentiment, il y a une part de simplification: le passé est passé, on en garde une ligne dominante en oubliant les conflits ou le fait qu'à chaque époque le futur a été opaque ou en tout cas incertain. C'est, un effet de lointain de penser que l'enseignement de l'église unifiait la pensée du «Moyen-Âge », que le XVII e siècle était tout «classique » ou que la croyance au progrès régnait sans problème au XIX siècle finissant. Ce qui n'empêche pas que c'est l'éclatement de nos normes à nous que nous avons à affronter.

La Déclaration des Droits de l'Homme et du Citoyen, août 1789 (Paris, Musée Carnavalet).

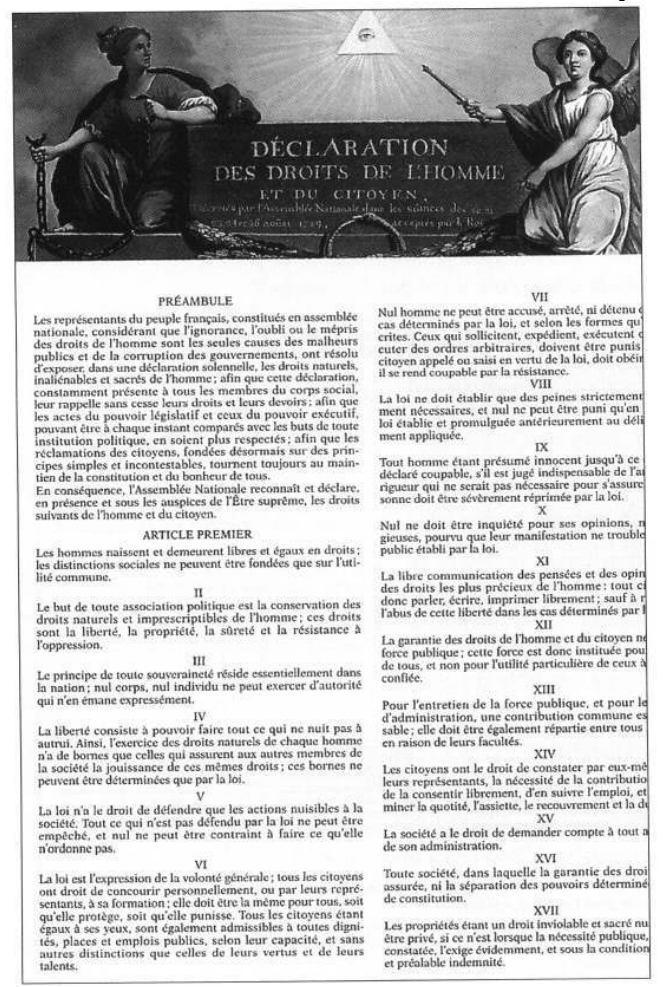

BENDJEBBAR A. (et al.) : Histoire Géographie Initiation Économique - $4^{\mathrm{e}}$ (Collection : Nouveauté 1992). Paris, Hatier, Avril, 1992, 351 p., p. 68.

7 Mon point de départ sera que maintenant comme «avant », il y a toujours une distance entre la position de principes et la justification de ces principes d'une part, la discussion sur leur applicabilité de l'autre.

8 Ainsi, un large accord peut se dessiner sur le fait que la pratique d'un sadisme raffiné aboutissant à d'horribles supplices n'est pas ce que demandent les parents aux enseignants à qui «ils confient leurs enfants ». Dès qu'on se demande pourquoi il en est 
ainsi, quel en est le «fondement ", les choses deviennent plus compliquées. Volonté de Dieu, opposition à la nature humaine, contradictions avec les règles de la «vie en société », réciprocité minimale du "ne fais pas à autrui ce que tu ne voudrais pas [...] ", sentiment de l'enfant comme sacré dans sa confiance et sa fragilité, dégoût ressenti à l'égard de telles pratiques, nécessité de refouler les tristes pulsions sous-jacentes à notre « mauvaise nature »...?

Et, encore, ici le consensus est-il fort.

Si l'on prend l'assertion des «droits de l'homme » en général, l'inquiétude augmente : les déclarations de principes s'accompagnent de justifications qui font problème ainsi dans la Déclaration des droits de l'homme et du citoyen de $1789^{1}$ : "les représentants du peuple français, constitués en Assemblée nationale, considérant que l'ignorance, l'oubli ou le mépris des droits de l'homme sont les seules causes des malheurs publics et de la corruption des gouvernements [...] ». On le voit, la justification est elle-même discutable, renvoie à un arrière-fond historique, est telle en tout cas que même si nous reconnaissons qu'elle dit quelque chose d'important, on ne pourrait la répéter telle quelle. Sans doute encore plus dans le cas de la déclaration universelle des droits de l'homme de 1948 article $1^{2}$ « Tous les être humains naissent libres et égaux en dignité et en droits. Ils sont doués de raison et de conscience et doivent agir les uns envers les autres dans un esprit de fraternité"».

Le moins qu'on puisse dire est qu' "être doué de raison et de conscience» est une assertion source de problèmes.

D'autant qu'on a pu noter qu'historiquement les premières positions associaient « droits de l'homme » et «droits du citoyen » et qu'il a justement fallu les violences spécifiques de notre époque pour que se pose la question des « droits de l'homme » indépendamment de ceux du citoyen. Ce qui renvoie d'ailleurs, comme le note Blandine Kriegel à une toute autre tradition ${ }^{3}$. La tradition citoyenne trouve ses racines dans la pensée athénienne, qui ne s'occupait guère de l'humanité en général, bien plus présente dans les monothéismes et dans le christianisme en particulier. Puis dans la tradition thomiste de limitation de la toute puissance divine par l'affirmation d'une nature humaine. Ceci repris à partir de l'actualité de la "question indienne» par Vitoria et Bartolomé de las Casas ou au contraire dans la proclamation des «droits de la conscience errante» par Bayle, le «fondement » devenant alors une nature inachevée plus qu'une « vraie nature ».

Ce qui peut nous laisser supposer que, justement, il fait partie de la nature des « principes » de n'apparaître que dans l'éclairage d'urgences et d'évidences différentes ou contradictoires...

14 Toujours est-il qu'à l'autre bout, si l'on peut dire, celui de l'application, chacun reconnaît au juge la possibilité d'interpréter la loi, en particulier de rechercher des circonstances atténuantes. Mais manifestement, on ne sait jamais très bien ce qu'il en est. De même que si on est d'accord sur la privation de liberté de tel criminel, les fonctions de mise à l'écart, de punition, d'empêcher de nuire, de réhabilitation sont des objets de discussions infinies. Comme il en est de même du moindre examen des fautes et des mérites de chacun d'entre nous.

15 Et sur de tels problèmes, il y a des évolutions, des évidences qui deviennent dominantes, mais pas à proprement parler des positions qui seraient « réfutées ».

16 L'exposé ici présenté va donc comporter une première partie, longue, rappelant quelques difficultés propres à l'espace de discussion. Une dernière partie brève qui cherche à préciser quelques caractéristiques positives de l'espace de discussion. Avec entre les deux 
une brève transition concernant l'impossibilité de concevoir un avenir radieux qui serait celui du règne du vrai scientifiquement établi ou de stigmatiser le non-vrai comme idéologie.

\section{De quelques difficultés de la relation de «transmission de la raison »}

\subsection{L'épaisseur de l'histoire}

17 La première serait sans doute celle qui provient du fait que nous avons été enfants avant que d'être hommes. Mais dans l'esprit cartésien, il s'agissait surtout de trouver une cause rendant compte du fait que nous ne sommes pas dans le vrai. De même que l'on peut attribuer à l'enfance passée ou toujours en nous tout ce qui fait qu'il y a des terreurs, des attachements, par exemple à la personne du sauveur paternel et «charismatique » alors que si nous étions comme dans les théories contractuelles des frères égaux, nous serions de plain pied dans la raison.

Par ailleurs, notre naissance dans tel lieu, dans telle famille manifeste primordialement notre contingence. Nous n'avons jamais demandé à naître, ni à naître de tel sexe, avec tel statut social dans tel groupe à tel moment... La culture, les principes, les interdits nous viennent d'abord de l'extérieur, comme de purs faits et c'est seulement dans un mouvement second que nous pouvons réfléchir, reconnaître que nos ancêtres ont pu se tromper, accepter, refuser ou modifier les traditions diverses qui nous sont transmises (car personne n'a une seule culture simple et univoque).

Un troisième aspect tout aussi fondamental serait que chaque génération redécouvre le monde, mais ne peut le redécouvrir que selon des modalités au minimum un peu différentes de celles des générations précédentes. On peut prendre l'exemple des objets techniques, nouveautés pour ceux qui les ont vu apparaître qui vont de soi sans être datées pour l'enfant. La lumière électrique, le téléphone, la télévision, l'Internet ne sont pas successifs pour l'enfant. Plus généralement, les «nouveaux » ne peuvent partager ni nos évidences ni nos problèmes. C'est plutôt nous qui sommes en situation de changer d'horizon de sens à leur contact. Nous avons été formés à trouver normal d'avoir un métier, à définir l'homme par le travail. C'était, si l'on peut dire, la valeur commune de la « culture bourgeoise » et de la "culture prolétarienne », le "droit à la paresse » restant assurément marginalisé.

Mais de même que nous ne partageons qu'avec effort culturel le sentiment de ceux qui ont vécu les tranchées de 1914-18, le devenir du fascisme ou du communisme entre 1940 et 1980 sont des objets culturels (relativement) lointains pour ceux qui sont nés dans la dernière décennie.

21 Il faudrait ajouter que, bien sûr, nous pouvons essayer d'introduire une relation de raison et de discussion à l'intérieur de l'institution scolaire. Mais le juge, le commissaire de police ou tout simplement le parent ou l'enseignant qui « disent la raison», sont, qu'ils le veuillent ou non, en position de force par rapport aux plus jeunes et leur raison est toujours potentiellement "musclée », un peu comme lorsque c'est le représentant des Etats-Unis qui explique aux gouvernants des petites républiques d'Amérique centrale les bienfaits des droits de l'homme et de la démocratie. Il y a, au moins, un «malaise " potentiel. 


\subsection{Le difficile n'a pas de limites} infiniment plus vastes que les limites de ce qui peut être fait. Il y a, Piaget y a beaucoup insisté, une logique stricte de l'action, où chaque opération modifie ce qu'on peut faire en même temps ou après. On ne peut en même temps faire et défaire, avancer et reculer, mettre ensemble n'importe quoi. Alors qu'il fait partie par exemple de la coordination linguistique de pouvoir mettre ensemble n'importe quoi dans la réalité symbolique (au sens de montrée seulement par le langage et non par présentation directe). Pour prendre un exemple récent, on a entendu un homme politique français renommé, discutant de la possibilité de réévaluer les fusillés du Chemin des Dames, dire «et pourquoi pas les Waffen SS? ». On peut tout mélanger dans le discours, beaucoup moins, par exemple, s'il s'agit de faire la cuisine ou de faire tenir un échafaudage.

Plus spécifiquement des auteurs comme Grize $e^{4}$ ont beaucoup insisté sur le fait que la logique «naturelle» (non au sens de donné par la nature, mais de celle réellement utilisée par opposition à la logique fabriquée ad hoc dans les espaces de pensée logique) consistait à fabriquer des entours, à constituer des points de vue. Certes, certains de ces entours, de ces cadres peuvent faire rire. Reste que le même fait sur lequel on peut s'accorder comme fait peut être replacé dans des ensembles variés.

Un exemple, assurément improbable. En $1995^{5} \mathrm{~J}$. Tibéri, accusé d'avoir fait profiter certains de ses amis et proches de loyers des H.L.M. de la ville de Paris particulièrement favorables, écrit «Nous ne pouvons pratiquer pour ces logements les prix du marché car nous ne pouvons pas entrer dans la spéculation immobilière ». On peut certes s'interroger sur la place de J. Tibéri comme défenseur des loyers bas contre les vilains spéculateurs, reste que le mouvement est possible.

De la même façon, je lis dans Le Monde du 12 Novembre 1998 sous la plume d'un avocat que je ne connais pas, Thierry Lévy: «Monsieur Roland Dumas: ne démissionnez pas. En résistant aux pressions, l'ancien ministre des affaires étrangères sert la cause de tous les accusés ". Ce qui constitue un déplacement inattendu de cadre par rapport à la doxa selon laquelle la fonction de président du Conseil constitutionnel exige qu'on ne puisse même pas être soupçonné d'indélicatesse.

Mais pour aller vers du plus complexe, si l'on suit la discussion actuelle sur la régularisation des "sans papiers ", on voit, me semble-t-il, ceux qui sont favorables à cette régularisation insister avant tout sur le nombre fini actuel de ceux qui se sont déclarés et qui se trouvent maintenant pris au piège de cette déclaration, en ajoutant, par ailleurs que les règles administratives ont évolué de façon restrictive. Au contraire, ceux qui s'y opposent, le premier ministre en tête, tiennent avant tout compte d'un avenir potentiel ouvert, sur l'effet d'appel d'air que, selon eux, cette régularisation globale ne manquerait pas de produire.

Cela ne signifie pas qu'on ne peut pas du tout discuter, mais qu'on ne peut calculer purement et simplement, qu'il y a bien plutôt contraste entre deux mondes, deux univers de référence différents, ce qui change la signification de ce qu'est le fait même de discuter.

Ou, pour prendre un autre exemple, Hannah Arendt ${ }^{6}$ revenant en Allemagne après la fin de la seconde guerre mondiale notait la difficulté qu'il y avait à discuter de la spécificité 
de ce qu'avait été la «solution finale » nazie au problème juif, les Allemands répondant, soit sur le mode " tous coupables » en évoquant les bombardements de civils (Dresde) ou Hiroshima et Nagasaki, soit au contraire " $" L^{\prime}$ Allemand moyen ne cherche pas les causes de la dernière guerre dans les actions du régime nazi, mais dans les événements qui ont conduit à l'expulsion d'Adam et Eve hors du paradis terrestre ». Mais ça ne concerne pas qu'eux. Qui d'entre nous est sûr qu'il construit «le bon cadre » pour penser telle violence ou telle injustice?

\subsection{Le difficile accord sur des définitions}

Ce à quoi il faut ajouter que les objets théoriques sur lesquels nous parlons sont des objets complexes, sur lesquels on peut toujours jeter un nouveau regard, ajouter une autre détermination.

On peut en effet s'interroger sur le fait qu'à l'heure actuelle, les discussions sur l'état et la démocratie partent toujours des définitions antiques, aristotéliciennes singulièrement ${ }^{8}$, cela malgré les différences évidentes de la situation des cités grecques, Athènes en particulier et de nos sociétés actuelles.

31 Ainsi quatre traits présents dans la problématique aristotélicienne continuent à faire sens pour nous ou en tout cas dans l'espace théorique de ceux qui ont droit à la parole. Tout d'abord, pour Aristote, la politeïa (que les Romains traduisent république [res publica]) concerne des citoyens libres et égaux par opposition aux esclaves. Le gouvernement s'y exerce par la loi et non par la force. Il a en vue l'intérêt général et non l'intérêt particulier. Enfin, il caractérise une communauté (koinonia) et non un peuple (ethos). Cette communauté pouvant s'accroître il y a naturalisation, affranchis, etc.

Date encore d'Athènes l'opposition entre gouvernement d'un, de plusieurs, de tous.

Naturellement la définition même du bien commun ou de ce que c'est qu'être égaux est elle-même lieu de nouveaux problèmes, mais il demeure que liberté, loi, bien commun, communauté, restent des références stables. Ce qui remet en cause l'historicisme absolu selon lequel chaque société sécréterait ses normes...

Reste que cette détermination stable ne suffit pas.

On peut lui opposer que la cité grecque avait d'autres caractéristiques négligées par cette définition «institutionnelle». D'abord, bien sûr, les dimensions qui font que chacun pouvait se rendre à l'Assemblée (ce qui d'ailleurs n'empêche pas que même alors chaque citoyen est égal en droit, pas égal effectivement dans la prise de parole à l'assemblée. Déjà là, il y en a qui parlent au nom des autres. Ensuite, comme on le rappelle souvent, il y a les exclusions, des esclaves, des femmes.

Et puis aussi l'évidence militaire, les citoyens étaient potentiellement soldats, ce que les citoyens actuels sont de moins en moins. Par parenthèse, face à l'apocalypse nucléaire ce sont peut-être maintenant les seuls militaires et leurs équipements sophistiqués qui ont quelques chances de s'en sortir.

Et puis aussi, il y a la dimension sacrée, de l'appartenance à une même religion. Et puis aussi tout ce qui unifiait le groupe dans sa différence face aux autres...

Et en ce qui nous concerne, on peut certes caractériser en première instance notre régime par la démocratie, par opposition par exemple à l'Etat français de 1940-45, ceci ne nous donne évidemment pas une image de son fonctionnement exact. 

rendent la vie sociale impossible et détruisent la citoyenneté au sens politique étroit alors que dans d'autres cas, c'est la vie politique citoyenne qui modifie l'excès d'inégalité caractéristique de la vie civile. Tout ceci ne pouvant se manifester que dans un espace de discussion, où la seule chose certaine est la nécessité de maintenir la possibilité de discuter, malgré l'impossibilité d'une hiérarchie univoque des valeurs, l'entrelacs des valeurs et des faits, les modifications des perspectives historiques (que l'on songe à l'évolution de nos évidences depuis cinquante ans sur le communisme ou la religion).

\subsection{Un exemple juridique.}

Dès qu'il y a discussion juridique se pose le problème des manques du droit, de l'évolution juridique, de l'opposition du droit international et du droit de tel pays, du conflit des exigences morales et du droit établi. Ce qui fait que, par exemple, dans le cas frappant de l'immunité à accorder ou non à Pinochet en tant qu'ancien chef d'Etat, on a vu deux des Lords qui se sont affrontés conclure, à partir de points de départ proches, l'un à 
l'immunité, l'autre contre. On ne peut résumer ici ces textes (extraits dans Le Monde du 27 novembre 1998). Je note que l'un d'entre eux rappelle d'abord l'origine de l'immunité des chefs d'Etat à partir de l'immunité des ambassadeurs, justification claire du début du droit contre la force : on ne tue pas l'ennemi qui vient négocier. Ensuite, cette immunité s'étend aux chefs d'Etat, à leur famille, à leurs domestiques, puis aux anciens chefs d'Etat. Ce qui entraîne le problème posé aux Lords. En gros, celui qui est pour l'immunité comme celui qui est contre reconnaissent tous deux l'évolution du droit international et le fait que torturer nationaux ou étrangers ne fait pas partie des attributions du chef d'Etat comme tel. Mais l'un considère que dans l'état actuel des choses, il n'y a pas d'instance supranationale ultime et d'autre part que les limites exactes des fonctions de l'homme d'Etat ne sont pas fixées. Enfin qu'«il est clair que le droit international n'admet pas qu'il revienne à un chef d'Etat de commettre la torture ou le génocide. Mais le fait qu'en accomplissant d'autres fonctions, le chef d'Etat commette un acte illégal ne signifie pas qu'il doive être tenu comme n'accomplissant pas son office. Si c'était le cas, l'immunité à l'égard des actes criminels serait vidée de l'essentiel de son contenu». Ce qui est «logique»: l'immunité pour les innocents n'a que peu d'intérêt.

Pour son adversaire « Tous les Etats désapprouvent l'usage de la torture, qu'ils jugent haïsable, même si de temps à autres certains y ont encore recours... La loi internationale reconnaît, bien entendu, que les fonctions d'un chef d'Etat peuvent comporter des activités détestables, illégales même, selon la loi de son propre Etat ou celle d'autres Etats. Mais la loi internationale indique clairement que certains types de conduite, dont la torture et la prise d'otage, ne sont acceptables de la part de quiconque ». Ce qui permet la référence analogique à Nuremberg. "Celui qui viole les lois de la guerre ne peut obtenir l'immunité en agissant conformément à l'autorité de l'Etat si l'Etat, en autorisant ces actes sort de ses compétences selon la loi internationale ». Bien entendu, je ne suis pas juriste et je ne rends compte de toute façon que d'une toute petite partie de l'argumentation. Reste qu'on voit bien sur une base commune la possibilité de deux éclairages différents. Pour reprendre le terme de Grize, il s'agit de construire des faisceaux d'objets, non de raisonner. On raisonne pour embarrasser l'adversaire, mais pas pour résoudre positivement un problème.

\section{Deux voies discutables}

On pourrait à l'espace de discussion, qu'il s'agisse de celui du "café du commerce ", du café philosophique ou de la classe, opposer la technicité croissante de notre monde et la nécessité de s'en remettre à des techniciens. Mais d'abord, sans nier qu'il y ait des problèmes techniques qui ne se résolvent pas par la discussion, c'est encore un problème de discussion de savoir ce qui va être le domaine spécifique des techniciens. Surtout, il est évident que, sans cesse, les experts ont une façon violente, pas purement technique de nous présenter les données du problème.

L'exemple le plus manifeste est celui de l'énergie atomique. Jusqu'à un passé récent, les Français de droite comme de gauche ont vécu dans l'évidence qu'il s'agissait de la seule source d'une énergie peu coûteuse, condition de l'autonomie du pays face aux aléas de la fourniture du pétrole. Le moins qu'on puisse dire est que cette belle évidence se fissure, modifiée en particulier par la remise en cause des espoirs liés aux surgénérateurs, aux problèmes de sécurité, à celui de la gestion des déchets, à celui des difficultés en termes de coût et de sécurité liées à la mise hors service des centrales qui ont fait leur temps. 

individuelles d'une part et la vérité universelle de l'autre, sur le modèle des mathématiques. Il me semble qu'on n'en est pas là, mais dans un espace où la particularité de chacun comme mode d'accentuation forcément variable pour un individu ou un groupe se modifie ou en tout cas change de signification dans le dialogue. Il ne 
s'agit pas de la décentration qui serait liée au seul fait de passer du particulier à l'universel. Mais de voir que la violence qui est en nous chaque fois que nous prétendons avoir raison se modifie lorsque notre point de vue se confronte à celui de l'autre. On peut s'accorder sur le fait qu'une certaine sécurité est une condition nécessaire d'une vie vivable. Mais pour l'un la sécurité est le plus important, pour l'autre courir des risques importe plus. On ne peut pas parler des «valeurs » comme telles, mais seulement en fonction des conditions où elles se manifestent et aussi des personnes qu'elles concernent. Pour faire vite, on ne peut pas justifier universellement une «échelle des valeurs ", mais on peut, c'est déjà beaucoup, relativiser son propre système d'évidences...

De la même façon, dans l'espace de discussion, il y a du déjà codé, du discours dominant (les médias sont là pour un coup). De même qu'il nous arrive à tous d'avoir le sentiment intellectuel que quelque chose cloche sans pouvoir l'expliciter.Ceci serait lié à la notion de raison comme surprise. Je m'aperçois que d'autres ne pensent pas comme moi ou que je pense tantôt ceci tantôt cela.

Ce mouvement prend du temps. Et le problème n'est pas d'arriver à une vérité définitive, mais de pouvoir faire ensemble des mouvements. Sans parler des mouvements que chacun fait à partir de ce dialogue quand il se retrouve dans la solitude du dialogue avec soi et/ou les autres fictifs ou « intériorisés ». Sans, par parenthèse, qu'il soit nécessaire de dire que l'accord avec soi importe plus que l'accord avec les autres. Il y a des cas où l'autre en soi fait peu d'objections. Même s'il est vrai que parfois aussi il y a des cas où le groupe est trop content d'être dans le consensus.

51 Face aux exigences du maintien de l'ordre et à celles de l'obligation de décider, l'espace de discussion est un espace de suspension qui suppose des conditions matérielles, un certain nombre d'interlocuteurs (ni trop ni trop peu), un lieu favorable où on obéit à des rituels par exemple d'écouter même ceux dont on peut penser a priori que ce qu'ils peuvent dire n'est pas très important et où, quoi qu'il en pense, le professeur ou plus généralement celui qui en sait (un peu) plus, n'a pas forcément raison contre les autres. Lieu aussi où le discours a « des hauts et des bas » : on ne voit pas de discussion qui serait une progression régulière vers « le vrai ».

52 L'exemple improbable qui a fait de la chambre des Lords un modèle de débat démocratique nous a tous frappés.C'est la relation entre discussion et "vérité » qui apparaît dans une citation de Montesquieu dans le numéro du Courrier de l'Unesco ${ }^{12}$ intitulé Droits humains : une conquête inachevée. (La citation ne comporte pas de renvoi exact et mon inculture ne m'a pas permis de la reconstituer). «Dans une nation libre, il est très souvent indifférent que les particuliers raisonnent bien ou mal : il suffit qu'ils raisonnent. De là sort la liberté qui garantit les effets de ces mêmes raisonnements ».

D'autre part, cet espace de discussion suppose un minimum d'accord sur ce qu'on ne va pas discuter, aussi sur le fait que ça vaut la peine de discuter ensemble, que ce n'est pas une pure contrainte. Mais inversement, il faut que les différences puissent s'exprimer, que celui dont l'opinion est potentiellement scandaleuse, qui pense que ce n'est pas vrai que tous les hommes sont égaux ou qu'il faut respecter ses parents puisse parler.

Deux autres caractéristiques. D'abord, c'est un espace fragile, la violence verbale ou autre peut toujours réapparaitre, mais aussi l'argument d'autorité ou l'ennui ou la dictature de l'habile, de celui qui sait se faire valoir. Sans oublier la limite que constitue le recours invérifiable au moins dans le moment aux statistiques ou aux témoignages. 

notre ministre (Libération du $1^{\mathrm{er}}$ décembre 1998). Expliquant qu'il fallait réformer les IUFM, le ministre a ajouté que "des cours sur la drogue, la violence, la situation dans les quartiers difficiles et la morale civique sont plus importants que la philosophie ». Naturellement, il peut y avoir une certaine injustice à couper ces propos d'un contexte plus large. C'est même un exemple de la morale de la discussion. Reste qu'il est à craindre qu'on (ministre ou autre) puisse croire qu'il suffirait de faire cours sur ces sujets difficiles pour que tout aille mieux. Or il me semble qu'il est impossible ici de «faire simple », de " dire seulement les principes ». On ne peut pas couper le problème de la drogue de celui des boissons alcoolisées des générations précédentes, non plus que de l'absence de perspectives, d'avenir, du chômage, d'un entourage de consommation ostentatoire comme modèle culturel dominant, d'une justice et d'une police qui s'en prennent davantage aux consommateurs ou aux petits revendeurs qu'aux "gros» etc. Mais aussi de la modification des formes de l'autorité familiale, de la modification du rôle de l'école qui ne peut plus être (si elle l'a jamais été) la principale source d'acculturation.Pas plus qu'on ne peut « enseigner la morale » sans faire le détour par les sociétés de bandits, les mafias qui sont les lieux sociaux où règne la règle morale la plus forte, ce qui est vieux comme Platon...

59 Second point. Cet espace long est d'autant plus nécessaire que nous savons qu'il n'est pas possible que tout le monde soit pareil, parte du même point et arrive au même point, que la discussion est toujours à reprendre...Et cela dans un double contexte d'urgence ${ }^{13}$ et d'affect. D'urgence: certes, on peut discuter "pour le plaisir». Reste qu'il y a des horizons, des menaces. Nous sommes entre un renouveau de pensée raciste, hargneuse, autoritaire et une dictature des décisions financières-techniques.D'affect : ce qui nous met en mouvement, c'est la peur, l'enthousiasme, la révolte. Le but n'est pas d'avoir une pensée désaffectivisée. De même qu'il y a forcément un lieu d'où on part, si l'on veut des préjugés. Le but est bien plutôt de savoir faire quelques mouvements de pensée à partir de là... de même qu'une opinion, un préjugé, reliés aux points de vue de l'autre ou à des mouvements, deviennent points de vue.

D'où la tension perpétuelle entre la prétention à l'universalité des principes et les conditions concrètes de leur énonciation et de leur application. Avec la contrainte de 
l'urgence, du proche et du lointain : les problèmes des droits de l'homme ne se posent pas « en général ». Le problème aussi que l'on peut, peut-être, s'accorder sur des principes. Mais que la discussion reprend dès qu'il est question de les fonder ou au moins de les justifier. De même de les appliquer, en fonction des urgences, ou des contradictions qui peuvent naitre par exemple entre libre choix et obligation d'égalisation. Que l'on pense par exemple aux quotas de telle ou telle catégorie parmi les enseignants ou les étudiants de l'université. Mais il faudrait avant tout ajouter que la nécessité de discuter, d'articuler différemment ne saurait être une objection de principe contre l'exercice de la raison. Une raison aboutissant à l'accord absolu de tous serait un fantasme terrorisant...

\section{NOTES}

1. DELMAS-MARTY \& LUCAS DE LAYSSAC C. : Libertés et droits fondamentaux, Introduction, textes et commentaires. Paris, Seuil-Points, 1996, p. 4

2. Ibid., p. 136.

3. KRIEGEL B. : Philosophie de la république. Paris, Plon, 1998, p. 128-133.

4. GRIZE J.-B. : Logique et langage. Ophrys, 1990.

5. FRANÇOIS F. : «Discuter pour quoi faire ? Ou la morale, le dire et le reste. ", in - DELAMOTTE

R., FRANÇOIS F. \& PORCHER L.: Langage, éthique, éducation, perspectives croisées. Rouen, Publications de l'Université de Rouen, 1997.

6. ARENDT H. : Penser l'événement (tr. fr). Paris, Belin, 1989.

7. Ibid., p. 54.

8. $c f$., Note 2 .

9. Cf., Note 5, p. 241.

10. RICOEUR P. : L'idéologie et l'utopie. Paris, Seuil, 1997.

11. Ibid., p. 21.

12. OUVR. COLLECTIF: «Droits humains: une conquête inachevée», in Le Courrier de l'UNESCO. Paris, UNESCO, Octobre 1998

13. FAYE J.-P : Faut-il défendre la République? Pleins feux, 1997.

\section{RÉSUMÉS}

Plus sans doute que dans les autres matières, le rôle de l'enseignant, en matière civique, est important. Certes, il sait des faits, connaît des penseurs que les élèves ne connaissent pas. Mais il n'est pas en situation de leur dire : «apprenez à penser: suivez mon exemple». D'où la nécessité de problématiser la question des savoirs et du langage.

The teacher's role in matters of civic education is important, undoubtedly more so than in other disciplines. It is certainly true, that he knows certain facts, is familiar with certain great thinkers 
with which the student are not. Yet, he is in no position to tell them: "To learn to think, think as I do". Therein, the need to define the problem of the issue of knowledge and language.

INDEX

Mots-clés : communauté de discours, philosophie, valeur morale

Keywords : community of discourse, moral value, philosophy

\section{AUTEUR}

\section{FRÉDÉRIC FRANÇOIS}

Professeur émérite, Université de paris $\mathrm{V}$ 